\title{
A Belongingness Principle of Motion Perception
}

\author{
Joseph C. DiVita \\ Naval Submarine Medical Research Laboratory
}

\author{
Irvin Rock \\ University of California, Berkeley
}

\begin{abstract}
Four experiments are reported that investigated the role of the perceived coplanarity of a moving target with respect to a frame of reference in the third dimension on the perceived path of that target. When a target dot and small moving frame appeared coplanar, the dot's perceived trajectory was governed entirely by its changing position relative to the moving frame. However, when the target and a large stationary frame appeared in a different plane than the small moving frame, the motion of the dot was seen independently of the moving frame. The results support a belongingness principle of motion perception: The displacement of an object relative to a frame of reference to which it belongs governs its perceived path of motion.
\end{abstract}

One of the important contributions Gestalt psychologists made to visual science was identifying stimulus relationships that determined perceptual phenomena. For example, the perceived lightness of a surface was held to be a function of the ratio between its luminance and the luminance of surrounding surfaces (Wallach, 1948). The perceived velocity of an object was not a function of physical or retinal velocity but rather was governed by the relative rate at which the object traversed a surrounding frame of reference (Brown, 1931/1965). The perceived orientation of a rod was influenced by the relationship between the rod and the surrounding frame (Asch \& Witkin, 1948a, 1948b), and the perceived motion of an object was determined by the relative displacement between the object and a nearby frame of reference (Duncker, 1929). In these examples, it was the relative, as opposed to the absolute, proximal information that governed perception. Insofar as these relationships remained invariant under conditions of changing absolute stimulation, the Gestalt psychologists claimed these relationships accounted for perceptual constancy (Wallach, 1948, 1976).

Modem research has continued to reveal stimulus relationships that determine our perceptual experience. For example, the perceived size of an object was found to be

Joseph C. DiVita, Vision Department, Naval Submarine Medical Research Laboratory, Groton, Connecticut; Irvin Rock, Department of Psychology, University of California, Berkeley.

The first draft of this article was prepared by Joseph C. DiVita and Irvin Rock. The final draft was submitted shortly after the death of Irvin Rock in the summer of 1995 . Final revisions were made by Joseph C. DiVita.

The views expressed in this article are those of the authors and do not necessarily reflect the official policy or position of the U.S. Department of the Navy, the U.S. Department of Defense, or the U.S. Government.

The data from Experiments 1, 2, and 3 were presented in a paper at the annual meeting of the Association for Research in Vision and Ophthalmology, Sarasota, Florida, in May 1993.

Correspondence concerning this article should be addressed to Joseph C. DiVita, Vision Department, Naval Submarine Medical Research Laboratory, Box 900 NAVSUBASE NLON, Groton, Connecticut 06349-5900. Electronic mail may be sent via Milnet to divita@nsmrl.navy.mil. influenced by the relative proportion of the object's height to the height of nearby objects in the visual field (Rock \& Ebenholtz, 1959). Statistical relationships between neighboring elements in patterns have been shown to underlie texture perception (Julesz, 1975). To account for why the perceived trajectories of elements in kinematic displays are usually different from their physical trajectories, Johansson (1973, 1975 ) proposed a perceptual vector analysis. In Johansson's analysis, the absolute motion of an element is decomposed into common and relative components. The common component refers to the motion of the entire configuration relative to the observer. The relative component refers to the motion of elements with respect to each other.

An example given by Johansson (1973) is illustrated in Figure 1A. In this display, the top and bottom dots move horizontally back and forth in phase while the middle dot moves along a diagonal path. The motion of the middle dot is such that it always remains in line with the top and bottom dots, that is, its horizontal position does not change with respect to these dots. Johansson proposed that all three dots share a common horizontal component of motion. Once this horizontal motion component is subtracted from the diagonal motion path of the middle dot, a vertical component remains (see Figure 1B). When viewing this display, observers report seeing all three dots moving as a group horizontally back and forth and the middle dot moving vertically up and down between the top and bottom dots (see Figure 1C).

Current research entails increasingly sophisticated analyses of the relationships inherent in the visual stimulus. The search for more complex, or higher order, relationships has been driven, in part, by the ecological theory of perception (Gibson, 1979), which contends that certain relationships contain invariant information about the environment. In the ecological approach, invariance has superseded the Gestalt notion of constancy.

In general, relationally determined perceptual phenomena have been taken as evidence against a cognitive or computational view of perception. Relationships in the stimulus can be registered by an active observer and do not seem to necessitate inferential or problem-solving processes. However, the search for higher order invariants has proved 


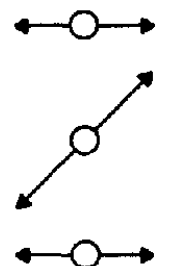

A

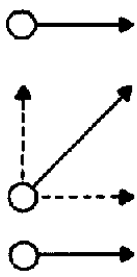

B

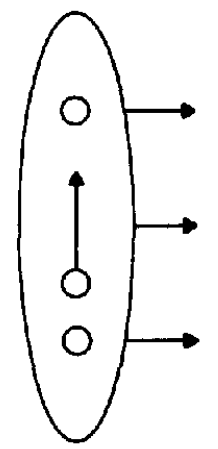

C

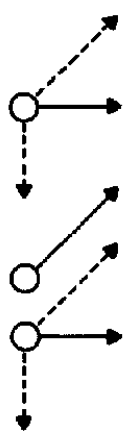

D

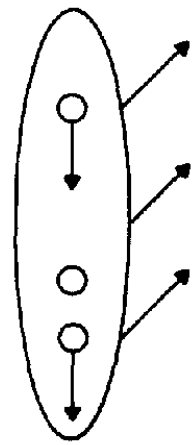

E

Figure 1. An example of perceptual vector analysis. A: The arrows represent the physical path of the dots. B: The diagonal motion path of the middle dot is decomposed into vertical and horizontal components. C: The percept associated with the vector analysis in B. D: An alternative decomposition in which the horizontal motion paths are decomposed into diagonal and vertical components. E: The percept associated with the vector analysis in D.

elusive, and despite the computational sophistication of current analyses, the debate still continues among vision researchers as to whether the information contained in the proximal stimulus is ambiguous (Cutting, 1986; LonguetHiggins \& Prazdny, 1984; Marr, 1982). In addition, several experiments that reexamined relationally determined perceptual effects have demonstrated that inferential processes may have been governing these phenomena.

For example, Gilchrist $(1977,1979)$ demonstrated that the perceived lightness of a surface is governed by the luminance ratios within a perceived plane and not necessarily the luminance ratios of adjacent retinal areas. Thus, the 3-D layout of surfaces must be taken into account before a ratio principle is applied. Rock, Auster, Schiffman, and Wheeler (1980) demonstrated that the amount of motion induced on a stationary dot is a function of the misperception of the motion of a frame of reference with respect to the observer (egocentric motion) and that the sum total of the perceived motion of the dot and frame together must account for all of the relative displacement between these two objects. A similar explanation has been given for the perceived orientation of a rod surrounded by a tilted frame (DiLorenzo \& Rock, 1982).

With respect to motion perception, several investigators have pointed out an inherent ambiguity in Johansson's $(1973,1975)$ perceptual vector analysis (Börjesson \& von Hofsten, 1972; Cutting \& Proffitt, 1982; Shum \& Wolford, 1983). The decomposition is not unique, and in fact, an infinite number of decompositions are possible. For example, in Johansson's analysis of Figure $1 \mathrm{~A}$, the common component is obtained by projecting the diagonal vector, the motion path of the middle dot, onto the horizontal vectors, the motion paths of the top and bottom dots. Thus, the diagonal vector is analyzed into horizontal and vertical components. However, one could project each of the horizontal vectors onto the diagonal vector and, in turn, call the diagonal vector the common motion. The horizontal vectors in this analysis are expressed in terms of diagonal and vertical components (see Figure 1D). Shum and Wolford
(1983) used this specific example to point out the inherent ambiguity of the term common motion. If the visual system performed this analysis, the three dots of Figure 1 would be perceived as moving obliquely as a group while the top and bottom dots moved up and down and maintained a constant vertical separation between each other (see Figure 1E). Although this outcome is logically possible, observers never report seeing it. Thus, the visual system prefers certain solutions over others, and various investigators have proposed different rules to account for these preferences.

For example, Bb̈rjesson and von Hofsten (1972) proposed a decomposition rule which states that the common motion is chosen so that the residual motions vectorially sum to zero. Restle (1979) adapted Leeuwenberg's (1978) coding theory to decompose simple harmonic motions with five parameters. The number of parameters needed to specify a motion was the "information load" of the decomposition code. Perceived motion was found to be in accordance with the code that minimized information load (i.e., the code that reduced the number of parameters needed to specify the motion). Cutting and Proffitt (1982) discussed two types of decomposition strategies: those that first extract the common motion, leaving the relative motion as the residual, and those that first extract the relative motion and leave the common motion as the residual. They proposed that the minimization of both common and relative motions proceeded simultaneously and that perception was determined by the process that first reached completion. (This generally favored relative motion.)

\section{Scope of the Present Study}

Our purpose in this research was to demonstrate that the perceived motion of an object in the vicinity of a moving frame of reference is based on a hierarchical organization in which the object's motion is governed by its relation to the frame and the frame's motion is governed by its relation to the observer. The object is seen as belonging to the frame and thus also partakes of the frame's motion. We further 
claim that in our displays, it is only stimulus relations within a perceived frame that govern the outcome.

A typical induced-motion display consists of a luminous dot surrounded by the outline of a luminous rectangular frame (see Figure 2). The frame and dot are the only visible objects in an otherwise totally dark room. The frame is made to move horizontally at a speed below the subject-relative threshold for motion. Under these conditions observers report that the frame appears stationary whereas the dot appears to move. If the frame is made to move a little faster, at or just above the subject-relative threshold for motion, the observer may report that both the dot and frame appear to move in opposite directions.

Suppose we modified this induced-motion display as follows: The frame now moves horizontally back and forth at a speed clearly above the subject-relative threshold for motion, and the dot moves vertically. The dot's vertical motion always remains enclosed within the frame, and the motions of the dot and of the frame are in phase, that is, both the dot and the frame change their direction of motion at the

\section{INDUCED MOTION}
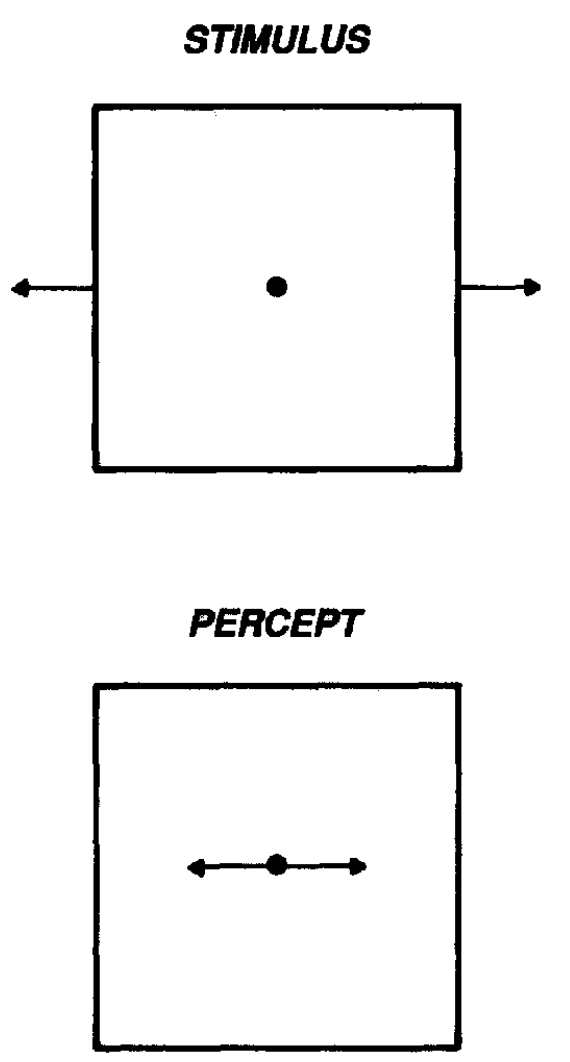

Figure 2. Top: A typical induced-motion display. A luminous outline of a rectangular frame moves horizontally back and forth below the subject-relative threshold for motion. The dot within the frame is stationary. Bottom: What observers typically perceive when viewing the top display. The frame appears stationary and the dot appears to move.
DIAGONAL EFFECT

STMULUS

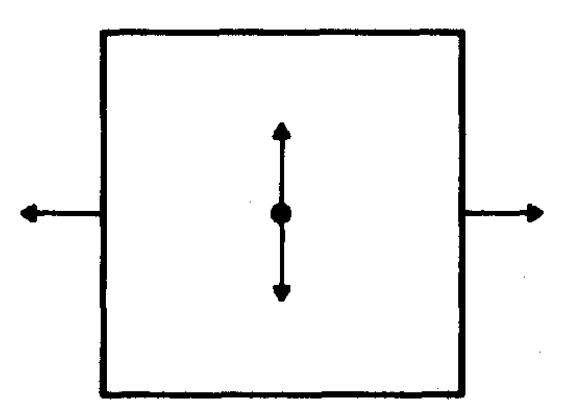

PERCEPT

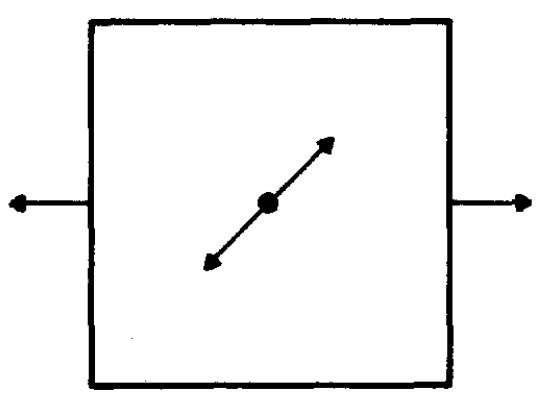

Figure 3. Top: Stimulus for the diagonal effect. Arrows indicate the direction and path of motion. Both dot and frame move well above the subject-relative threshold for motion. Bottom: Arrows denote the perceived paths of motion of the dot and frame.

same time (see Figure 3). Under these conditions, the dot is seen to be moving diagonally. We refer to this as the diagonal effect. It was first demonstrated by Wallach, Bacon, and Schulman (1978).

An explanation that is often given of the diagonal effect is that the dot undergoes induced motion horizontally. The induced horizontal component of motion sums vectorially with the actual vertical component of motion to produce the dot's diagonal trajectory (see Figure 4). The vector addition involved in the induced-motion explanation is equivalent to a vector analysis that subtracts the horizontal motion of the frame from the dot's vertical motion (assuming that the dot partakes of the frame's motion); however, the underlying explanation of the effect is different.

There are several reasons to reject the induced-motion explanation. In general, the diagonal effect is robust, whereas induced motion is fragile and often unobtainable under conditions that do not alter the diagonal effect. For example, the diagonal effect is obtained even if other objects are visible in the field; in particular, a stationary frame that surrounds the moving frame has no effect on the dot's perceived diagonal motion, but the presence of a stationary 
frame eliminates the induced-motion effect (Brosgole, 1968). The diagonal effect is also obtainable when the frame moves at rapid speeds, whereas induced motion is often not reported at these high frame speeds (Brosgole, 1968; Duncker, 1929; Rock et al., 1980). Last, for the inducedmotion effect, we contend that the motion of the frame is often misperceived, that is, underestimated or not perceived at all (Rock et al., 1980), but we do not believe this to be the case in the diagonal effect.

We believe our experiments demonstrate an alternative explanation to the diagonal effect. Our explanation is that the dot is perceived as belonging to the frame and partaking of the frame's motion. Of course, in the laboratory, the dot and frame are entirely independent objects. But we can imagine a more realistic situation that produces the same proximal stimulus and one in which the dot's motion is not independent of the frame's motion.

Suppose the frame is a rectangular surface that is moving horizontally and the dot is a self-propelled object moving obliquely along the rectangular surface (see Figure 5). If the horizontal component of the dot's motion matched that of the surface but in the opposite direction, then the dot's motion on the retina would be vertical-just as it is for the diagonal effect. One example of this is an insect walking obliquely along an animal as the animal moves horizontally. Even if the horizontal components do not match exactly, the motion of the dot on the retina is the result of both its motion and the motion of the surface. The direction of this retinal path is not the same as the direction of the path the dot travels along the surface. Yet we would not be surprised if we perceived the dot moving in the direction in which it is moving along the surface and not in the direction its image moves along the retina. Thus the dot would be perceived as moving obliquely across the surface as the surface moves horizontally across our visual field. But this is exactly how the dot and frame are perceived to move in the diagonal effect, which just entails the particular case of the horizontal component of the dot's motion equaling the frame's motion.

To demonstrate the hierarchical relationship between the dot and the moving frame, in our experiments we had the dot and the frame appear either in the same or different planes in depth. In general, if they appeared in the same plane, we

\section{STANDARD EXPLANATION OF DIAGONAL EFFECT}

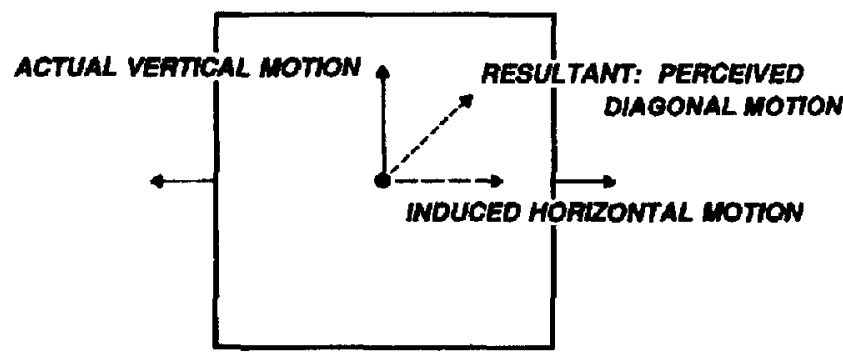

Figure 4. An explanation of the diagonal effect. The actual vertical motion and the induced horizontal motion sum vectorially to produce the diagonal effect.

\section{ALTERNATIVE EXPLANATION \\ OF THE DIAGONAL EFFECT}

DOT'S MOTION RELATIVE TO THE SURFACE

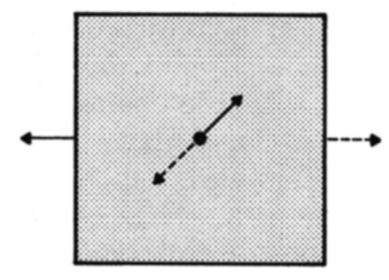

RESULTANT RETINAL IMAGE

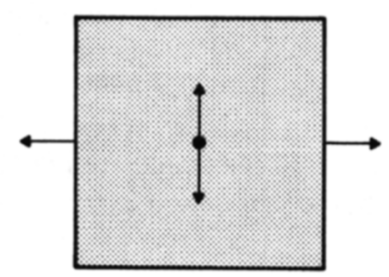

Figure 5. Top: Our explanation of the diagonal effect. The dot is physically attached to the moving frame and is moving diagonally across the frame. Bottom: The resultant stimulus is one where the dot moves vertically as the frame moves horizontally.

predicted a diagonal effect, but if they appeared in different planes, we predicted no diagonal effect would occur because the dot would not be perceived as being carried along by the frame, that is, as partaking of the frame's motion.

In terms of a vector analysis, our explanation is equivalent to subtracting the horizontal motion of the frame from the vertical motion of the dot. The problem that our research raises is, when does the visual system carry out this subtraction? Given the ambiguity of determining common motion in a kinematic display, the visual system must take into account other information-specifically, factors that pertain to grouping and belongingness-in order to determine if elements partake of a common motion. ${ }^{1}$ For example, in Figure 3, there is no information in the motion vectors per se that specifies whether or not the dot partakes of the frame's motion. Unlike the case in Figure 1, where the horizontal and diagonal vectors can be projected on one another in a nontrivial manner, the motion paths of the dot and the frame in Figure 3 are orthogonal to one another. The projection of one vector onto the other is zero. In this sense, the paths of the dot and the frame do not share a common motion, yet the dot can still be interpreted as partaking of the frame's motion. Thus, whether or not the dot partakes of the frame's motion is independent of the vectors associated with the absolute motion paths of the dot and the frame.

\footnotetext{
${ }^{1}$ A similar argument can be made for relative motion. That is, if one argues that it is relative motion that is extracted first by the visual system, then the problem still remains as to when, or under what conditions, the motion of one element is seen relative to the motion of another.
} 
There is nothing anomalous about the orthogonality of the motion paths in Figure 3 that allows belongingness to become a determining factor for the perceptual vector subtraction. Using displays similar to those pictured in Figure 1, we demonstrate in Experiment 3 that belongingness still plays a critical role in determining whether the vector subtraction is carried out by the visual system even if motion elements share a common component of motion. From our perspective, this is not surprising because the common motion is not uniquely specified.

From pilot experiments, we discovered that the separation in depth of the dot and the frame had little effect on the perceived path of the dot when there was no other visible region in the plane of the dot. We believe this effect is analogous to those concerning the perceived lightness and 3-D layout of surfaces. For example, depth organization can have a powerful effect on perceived lightness provided that there are other surfaces in the same plane as the test surface; however, depth organization has no effect on perceived lightness if the test surface is isolated in the plane (Gilchrist, 1977, 1979; Hochberg \& Beck, 1954). To overcome this difficulty, we placed a large stationary frame in the same plane as the dot.

Our displays use two frames and resemble the Gogel and Koslow (1972) demonstration of an adjacency principle for induced motion. However, there are good reasons for believing that our experiments cannot be explained by the adjacency principle and induced motion. We take these up in the General Discussion section.

\section{Experiment 1}

\section{Method}

Participants. Eight undergraduate students participated in the experiment for course credit. All observers had normal vision that did not require corrective lenses. The observers were naive as to the purpose of the study.

Apparatus. The displays for all of our experiments were generated on two Tektronix Type 5110 oscilloscopes with fastphosphor (P-15) radical-free screens. A special modification of the scope allowed independent $x, y$ channels to be manipulated onto the CRT screen. A specially built display control unit allowed multiple signals to be applied to each axis of the $x$ and $y$ channels and allowed ready manipulation of the amplitude and display traces. Signals were produced by Interstate Type F-43 function generators and an Apple computer with digital-analog converters.

Procedure. Observers were tested under darkroom conditions with their heads held stationary by a chin and head rest. The display, when viewed monocularly, appeared as a large stationary frame surrounding a smaller horizontally moving frame, within which a single dot moved vertically. The frames and the dot all appeared in the same plane, and the motions of the dot and the small frame were in phase (see Figure 6). When viewed binocularly, the frames appeared separated in depth. In the near plane, a small rectangular frame, $3.81 \times 3.56 \mathrm{~cm}$, moved back and forth horizontally at a rate well above the subject-relative threshold for motion. In the far plane, a pinpoint of light moved up and down within the outline of a large stationary rectangular frame, $11.43 \times$ $8.38 \mathrm{~cm}$. The length of the vertical path the dot traversed was 3.05 $\mathrm{cm}$. Depth was achieved with the use of a beam-splitting method (see Figure 7). This method required two CRTs placed orthogonal
EXPERIMENTI

BNOCULAA COMOMON

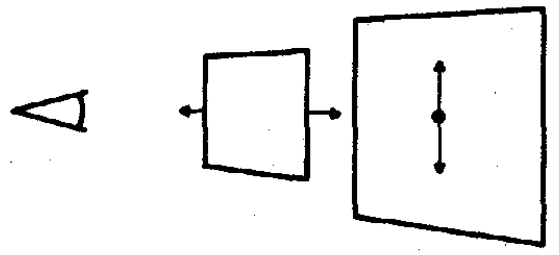

MOMOCULAR CONDTHON

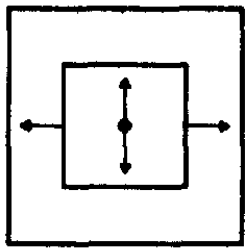

Figure 6. The display used in Experiment 1. Top: When viewed binocularly, the small frame appears in the near plane, and the large frame appears in the far plane. Arrows indicate the physical direction of motion. Bottom: When viewed monocularly, both planes are seen in the same plane. Arrows indicate the physical direction of motion.

to one another and a half-silvered mirror placed between them at a $45^{\circ}$ angle. The small moving frame was generated on one CRT, and the large stationary frame and moving dot were generated on the other. The optical distance of the small frame was $50.8 \mathrm{~cm}$. At this distance, the small frame subtended a visual angle of $4.3^{\circ} \times 4.0^{\circ}$. The optical distance of the far frame was $64.14 \mathrm{~cm}$, and it subtended a visual angle of $10.18^{\circ} \times 7: 48^{\circ}$. Thus, the depth between the near and far planes was approximately $13 \mathrm{~cm}$ (or roughly 5 in.).

The physical veiocities of the dot and the small frame varied sinusoidally; however, perceptually, this motion appeared linear. We used sinusoidal motion because linear motion produced an undesirable "bouncing" effect when the object instantly changed direction without changing speed. With sinusoidal motion, the object actually stopped before changing direction; however, this momentary pause was imperceptible. The average physical velocities of the dot and the small frame were $2.54 \mathrm{~cm} / \mathrm{s}$. This physical velocity produced retinal velocities of $2.86 \% / \mathrm{s}$ and $2.72 \% / \mathrm{s}$ for the frame and the dot, respectively.

Observers were tested in both monocular and binocular conditions. In the monocular condition, each eye was tested. The order of binocular and monocular viewing was counterbalanced across observers. Within the monocular condition, we also counterbalanced the order of which eye viewed the display first. The display was never visible to the observer while the viewing conditions were being changed. Observers were instructed to track the moving dot and verbally report its direction of motion. Afterwards, they matched the orientation of a luminous rod to the trajectory of the dot's motion. The luminous rod was to the side of the experimental display so that observers never saw the rod and the dot simultaneously. In general, there was no set duration of exposure to the display. Observers tracked the dot until they felt comfortable in making a match. Prior to viewing the moving dot in each condition, observers viewed the display with the dot stationary and were asked to describe the distances, from themselves, of the dot and the small and large frames. 


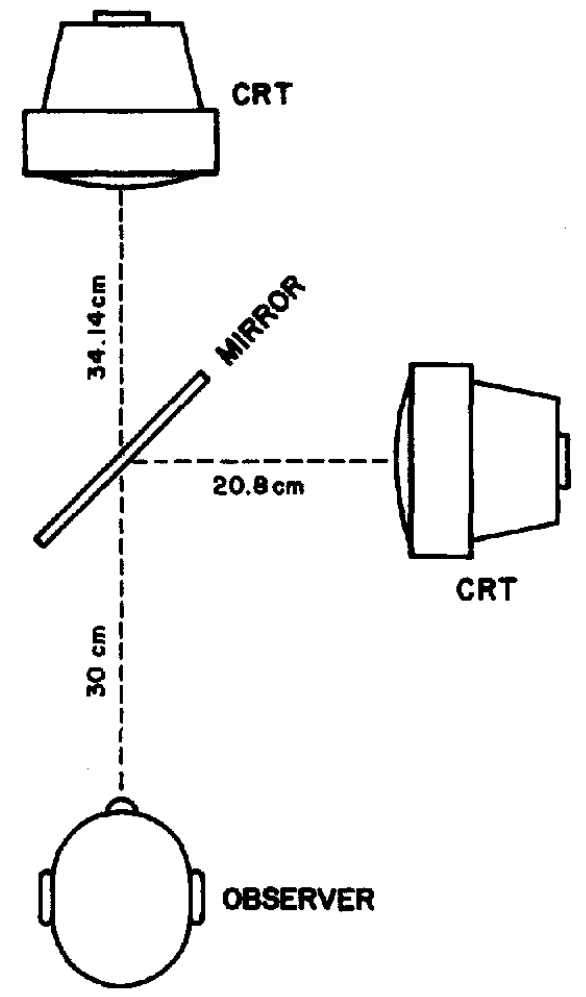

Figure 7. The physical arrangement of the CRTs and mirror used in the beam-splitting method to create the impression that the two frames were at different depths with respect to the observer.

Observers finished all the viewing conditions for the dual-frame display before they were tested on a single-frame display. We created the single-frame display by eliminating the large stationary frame of the original display. Thus, when observers viewed the single-frame display binocularly, the dot appeared behind the small frame with no other visible frame or object in the plane of the dot. When viewed monocularly, the frame and the dot appeared in the same plane. All observers were tested on the single-frame display in the same manner as they were tested on the dual-frame display.

\section{Results}

We first discuss the results for the dual-frame display (see Table 1). All observers correctly perceived the depth relations between the dot and the frames for both viewing conditions. When observers viewed the display monocularly, the perceived direction of the dot's motion was oblique and governed entirely by its relative position within the small frame. The median orientations of the luminous rod were $57.5^{\circ}$ and $50^{\circ}$ from the horizontal for the right and left eyes, respectively. (Medians were computed rather than means as a measure of central tendency because the number of observers in these experiments was relatively small and because outlier responses do not affect the median as they do the mean. However, the difference between medians and means was slight.) When the display was viewed binocularly, the dot was seen to move vertically by all observers, and all observers matched the rod to a $90^{\circ}$ orientation. The dot's motion was now seen relative to the large stationary
Table 1

Angle (in Degrees From the Horizontal) of the Perceived Direction of the Moving Dot for the Two-Frame Conditions of Experiment 1

\begin{tabular}{cccc}
\hline & \multicolumn{2}{c}{ Monocular } & \\
\cline { 2 - 3 } Observer & Right & Left & Binocular \\
\hline 1 & 73 & 60 & 90 \\
2 & 50 & 50 & 90 \\
3 & 55 & 50 & 90 \\
4 & 60 & 40 & 90 \\
5 & 55 & 50 & 90 \\
6 & 60 & 63 & 90 \\
7 & 54 & 41 & 90 \\
8 & 63 & 54 & 90 \\
$M d n$ & 57.5 & 50 & 90 \\
\hline
\end{tabular}

frame. Because the dot was not in the same plane as the small frame, its motion was seen as independent of the small frame's motion.

Results for the single-frame display are listed in Table 2. When viewed monocularly, the motion of the dot was again governed by its relative position within the small frame. When the display was viewed binocularly, all observers veridically perceived the dot behind the frame. However, observers reported that the dot moved along an oblique path. They verbally reported that there was no change in the direction of the moving dot between monocular and binocular viewing conditions. This is reflected in the matches made with the luminous rod. Medians of $57.5^{\circ}, 50^{\circ}$, and $50^{\circ}$ were obtained for the right eye, the left eye, and the binocular condition, respectively. Thus the perceived depth of the dot had no effect on its perceived path of motion.

This finding suggests that if there is no other frame or object in the plane of the moving dot, the dot's motion is seen relative to the moving frame by default. As mentioned in the introduction, we believe this effect is analogous to the case in which depth organization has no effect on perceived lightness if the test surface is isolated in the plane (Gilchrist, 1977, 1979).

Table 2

Angle (in Degrees From the Horizontal) of the Perceived Direction of the Moving Dot for the Single-Frame Conditions of Experiment 1

\begin{tabular}{cccc}
\hline & \multicolumn{2}{c}{ Monocular } & \\
\cline { 2 - 4 } Observer & Right & Left & Binocular \\
\hline 1 & 50 & 50 & 50 \\
2 & 50 & 50 & 50 \\
3 & 52 & 50 & 50 \\
4 & 65 & 45 & 50 \\
5 & 55 & 50 & 50 \\
6 & 60 & 65 & 65 \\
7 & 80 & 47 & 55 \\
8 & 70 & 40 & 55 \\
$M d n$ & 57.5 & 50 & 50 \\
\hline
\end{tabular}




\section{Experiment 2}

In Experiment 2, we modified the dual-frame display of Experiment 1 by adding a vertically moving dot to the near frame (see Figure 8). When viewed monocularly, the two dots moved vertically along the same path but $180^{\circ}$ out of phase. In accordance with a belongingness principle of motion perception, we predicted that the perceived trajectories of the dots would vary with monocular and binocular viewing.

When viewed monocularly, both dots and frames appeared in one plane. Insofar as both dots partook of the small frame's motion, we predicted that each dot would appear to be moving along a diagonal path and that their paths would crisscross, forming an $X$. However, unlike a typical diagonaleffect display in which there is only one dot, in this display, the objoct-relative motion of each dot with respect to the other was vertical. Thus there was a new source of objectrelative information that could lead observers to perceive the dots as moving vertically. Another possible interpretation is that the two dots could be perceived in depth as the endpoints of a vertical stick rotating about a horizontal axis. Wallach and O'Connell (1953) obtained a weak kinetic depth effect with the shadow of a $T$ figure rotating about a vertical axis, and Brorjesson and von Hofsten (1972) obtained reports of depth when two luminous dots traversed a horizontal motion path $180^{\circ}$ out of phase.

We predicted that when the display was viewed binocularly, only the dot in the near frame would partake of this frame's horizontal motion and as a consequence be seen to move diagonally. In contrast, the dot in the same plane as the far frame would now be independent of the small frame's motion and would be perceived as moving vertically.

\section{EXPEAITEMT}

BWOCULAR CONOTION

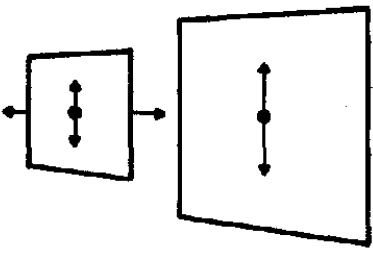

MOMOCULAR COMDTION

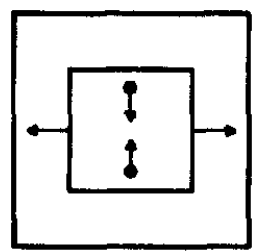

Figure 8. The display used in Experiment 2. Top: When viewed binocularly, the small frame appears in the near plane, and the large frame appears in the far plane. Arrows indicate the physical direction of motion of the dots and small frame. Bottom: When viewed monocularly, both frames are seen in the same plane. Arrows indicate the physical direction of motion.
Table 3

Angle (in Degrees From the Horizontal) of the Perceived Direction of the Moving Dots in Experiment 2

\begin{tabular}{cccccccc}
\hline \multicolumn{3}{c}{ Monocular condition } & & \multicolumn{3}{c}{ Binocular condition } \\
\cline { 1 - 2 } Observer & Near dot & Far dot & & Observer & Near dot & Far dot \\
\hline 1 & 51 & 45 & & 9 & 45 & 90 \\
2 & 50 & 52 & & 10 & 45 & 90 \\
3 & 54 & 52 & & 11 & 90 & 90 \\
4 & 90 & 90 & & 12 & 60 & 82 \\
5 & 45 & 45 & & 13 & 45 & 90 \\
6 & 45 & 45 & & 14 & 50 & 90 \\
7 & 50 & 45 & & 15 & 45 & 90 \\
8 & 52 & 45 & & 16 & 45 & 75 \\
$M d n$ & 50.5 & 45 & & Mdn & 45 & 90 \\
\hline
\end{tabular}

\section{Method}

Participants. Sixteen observers were paid for their participation. All observers had normal vision that did not require corrective lenses. The observers were naive as to the purpose of the study.

Procedure. The procedure for Experiment 2 was the same as that of Experiment 1 with a few minor changes. Half the observers were tested monocularly and half binocularly. In order to eliminate any blur in the image of the plane to which the observer was not accommodating at any moment, we had observers wear trial lens holders fitted with pinhole apertures. As in Experiment 1, observers tracked each dot and verbally reported the direction of motion of the tracked dot while viewing the display. Afterwards, they matched the perceived trajectories of the dots to the orientation of a rod. Across observers, the order of the tracked dots was counterbalanced.

\section{Results}

The data for Experiment 2 are listed in Table 3. Under monocular viewing conditions, 7 of the 8 observers saw the dots moving diagonally, whereas 1 observer saw both dots moving vertically. None of the observers reported a kinetic depth effect (that is, seeing the dots rotating in depth as if they were the endpoints of a vertical stick rotating about a horizontal axis). ${ }^{2}$ The median matches were $50.5^{\circ}$ and $45^{\circ}$ for the near and far dots, respectively. In accordance with our predictions for the binocular condition, 7 of the 8 observers reported that the near dot moved diagonally and the far dot moved vertically. Two of these 7 observers reported that the far dot's motion was "slightly" off the

\footnotetext{
${ }^{2}$ It is not surprising that a kinetic depth effect was not obtained under these conditions. The kinetic depth effect for the $T$.figure is weak, and a translating frame is a potential source of conficting depth information that is typically not presented in a kinetic depth effect display. Rock and Smith (1981) demonstrated that a static visible frame could be used to eliminate the kinetic depth effect, although the frame had to be perceived as occluding the endpoints of the rotating rod. (This is not the case in our display.) In both cases, it appears that a static or moving frame can provide the visual system with information that leads to an alternative interpretation of stimulus information typically associated with a kinetic depth effect.
} 


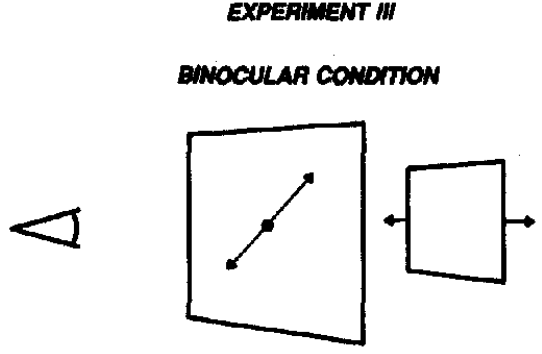

MONOCULAR CONOTTON

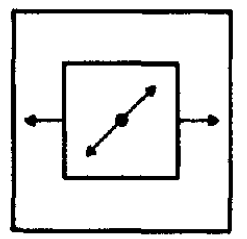

Figure 9. The display used in Experiment 3. Top: When viewed binocularly, the large frame appears in the near plane, and the small frame appears in the far plane. Arrows indicate the physical direction of motion of the dot and small frame. Bottom: When viewed monocularly, both frames are seen in the same plane. Arrows indicate the physical direction of motion.

vertical. The 1 remaining observer saw both dots as moving vertically. Median matches of $45^{\circ}$ and $90^{\circ}$ were obtained for the near and far dots, respectively.

\section{Experiment 3}

Hochberg and Fallon (1976) tested Johansson's (1973, 1975) perceptual vector analysis theory using a motion display similar to that in Figure 1. They found that the effect was not complete in that the motion of the middle dot was judged to be off the vertical. Shum and Wolford (1983) also found that the perceptual vector analysis was not complete in three-dot displays in which the center dot traversed an elliptical path and was flanked on each side by a vertically moving dot. In one of their displays, the two vertically moving dots were replaced by a vertically moving frame. On the basis of observers' judgments of the perceived trajectory of the middle dot, Shum and Wolford expressed the proportion of the common motion subtracted from the middle dot in terms of a parameter $k$ (where $k$ varied between 0 and 1 , and $k=1$ implied that all the motion of the frame of reference was subtracted, i.e., that the effect was complete). They obtained average $k$ values of 0.96 and 0.75 (Experiment 2) for displays with and without frames, respectively. They attributed the essentially complete effect when the frame was present to factors such as the adjacency principle and proximity.

In our Experiment 3, the dot and the stationary large frame were in the near plane, and the horizontally moving small frame was in the far plane. The dot moved diagonally and in phase with the motion of the small frame (see Figure 9). Thus, the dot did not change its horizontal position relative to the small frame. We predicted that when the display was viewed monocularly, the dot's motion would be perceived relative to the small moving frame; that is, the dot would be seen to be moving vertically. We refer to this as the vertical effect. We predicted that when the display was viewed binocularly, however, the dot would no longer appear in the same plane as the moving frame and would not partake of its motion. Instead, the dot's motion would be seen relative to the stationary frame, so the dot would then appear to be moving diagonally.

\section{Method}

Participants. Ten undergraduate observers participated in the experiment for course credit. All observers had normal vision that did not require corrective lenses. The observers were naive as to the purpose of the study.

Procedure. For one group of 4 observers, the optical distances of the CRTs were the same as those used in Experiments 1 and 2. Thus, this group was tested with great depth between the planes, on the order of $13 \mathrm{~cm}$ (approximately $5 \mathrm{in}$.). The visual angle of the large frame, now presented in the near plane, was $12.84^{\circ} \times 9.43^{\circ}$. Within the large frame, a dot traversed a $45^{\circ}$ diagonal path that was $4 \mathrm{~cm}$ in length (yielding a visual angle of $4.6^{\circ}$ ). The visual angle of the small frame, now in the far plane, was $3.40^{\circ} \times 3.18^{\circ}$.

A second group of 6 observers was tested with less depth, $3 \mathrm{~cm}$ (approximately $1 \mathrm{in}$.), between the planes. We accomplished this by shortening the optical distance of the far CRT from $64.14 \mathrm{~cm}$ to $53.8 \mathrm{~cm}$. At this distance, the small frame subtended a visual angle of $4.06^{\circ} \times 3.79^{\circ}$. This small depth eliminated any double imagery that may have arisen from tracking the dot in the binocular condition.

All observers viewed each display monocularly (with the right eye) and binocularly. Across observers, the order of monocular and binocular viewing was counterbalanced. For the binocular condition, none of the observers wore trial lens holders fitted with pinhole apertures. All other aspects of the procedure were the same as in Experiment 1.

\section{Results}

The results for the small- and large-depth displays were similar, so they have been grouped together. The results for almost all observers are in accordance with what a belongingness principle predicts (see Table 4). In the monocular

\section{Table 4}

Angle (in Degrees From the Horizontal) of the Perceived Direction of the Moving Dot in Experiment 3

\begin{tabular}{ccc}
\hline Observer & Monocular & Binocular \\
\hline 1 & 90 & 77 \\
2 & 90 & 60 \\
3 & 90 & 55 \\
4 & $90-80$ & 70 \\
5 & 90 & 45 \\
6 & 90 & 52 \\
7 & 90 & 40 \\
8 & 80 & 50 \\
9 & 90 & $45-90$ \\
10 & 90 & 60 \\
$M d n$ & 90 & 57.5 \\
\hline
\end{tabular}


condition, 8 observers perceived the dot to be moving vertically, and 2 observers perceived the dot's direction to be slightly off the $90^{\circ}$ vertical. The median match for the monocular condition was $90^{\circ}$.

In the binocular condition, 9 observers reported the dot to be moving along a diagonal path, and 1 observer reported that he could see the direction of the dot's path as either vertical or diagonal. This observer was tested on the small-depth display, where it is easy to note the position of the dot relative to the small frame even though they do not appear in the same plane. The median match for the binocular condition was $57.5^{\circ}$.

\section{Experiment 4}

From pilot experiments, we observed that induced motion did not occur at the relatively high frame speeds used in these displays (approximately $3 \%$ ). We designed Experiment 4 as a simple control condition to formally demonstrate this observation. The dot and frame were in the same plane, as is typical in induced-motion displays.

\section{Method}

Participants. Ten undergraduate observers participated in the experiment for course credit. All observers had normal or correctedto-normal vision. The observers were naive as to the purpose of the study.

Procedure. All observers were tested on an induced-motion display composed of a stationary dot surrounded by the outline of a rectangular frame. The dimensions, speed, and distance of the frame were the same as those used in Experiment 1 for the small frame. The dot was in the same plane as the frame. The horizontal motion of the frame was such that the dot always appeared inside the frame. Observers viewed the display monocularly and binocularly. (Under these conditions, the depth relationship between the dot and frame should not change as a function of viewing condition.) The order of the viewing conditions was counterbalanced across observers. Observers were instructed to report on the motion (or stationariness) of the dot.

\section{Results}

All observers in both the binocular and monocular viewing conditions reported that the dot was stationary.

\section{General Discussion}

We believe our experiments demonstrate that the diagonal and vertical effects cannot be explained by the adjacency principle and induced motion. Gogel and Koslow (1972) used two frames to demonstrate an adjacency principle of induced motion. In one of their dual-frame displays, the near and far frames moved in opposite directions below the subject-relative threshold for motion, and a stationary dot appeared in the near plane, the far plane, or at a distance halfway between the two. The adjacency principle states that as the phenomenal distance between the dot and a frame decreases, the contribution of that frame to the induced motion of the dot increases.

There are three reasons why we believe our experiments cannot be explained by the adjacency principle and induced motion:

1. The motion of our frame was well above the subjectrelative threshold for motion. When we used these speeds in a typical induced-motion display, our observers reported that the dot appeared stationary; that is, there was no induced motion (Experiment 4).

2. In an induced-motion display that uses only a single frame, increasing the phenomenal distance between the dot and the frame decreases the amount of induced motion of the dot (Gogel \& Koslow, 1971, 1972). However, in our single-frame displays, the separation in depth of the dot and frame had no effect on the perceived diagonal path of the $\operatorname{dot}^{3}$ (Experiment 1). This suggests a default condition in which the dot's motion is seen relative to the only frame of reference in the field, regardless of the depth relations between the dot and the frame.

3. Last, Gogel and Koslow (1972) reported that although the frame in the same plane as the dot dominated the induced-motion effect when two nonequidistant frames were presented, the noncoplanar frame substantially contributed to the dot's perceived motion. In our displays, the effect of the coplanar frame on the perceived trajectory of the dot was complete. The coplanarity of the dot and the frame determines, in an all-or-none fashion, whether the dot partakes of the frame's motion. In general, our results support this claim. We believe that the occasional exception to a complete effect was a consequence of the impoverished stimulus conditions of our display. There was little information in the stimulus to support the percept that the frame was a surface on which the dot was attached and that the dot was being carried along by the frame. In addition, we included other moving objects and frames, and it was relatively easy for an experienced observer to deduce the simple relationships between the moving objects. Yet despite these conditions, a large majority of our observers gave results in accordance with a complete belongingness (coplanarity) effect.

With respect to perceptual vector analysis, our experiments raise a broader issue. In the past, evidence that the visual system was performing a vector analysis was demonstrated in displays in which distance information and factors such as belongingness were held constant for all motion elements. For example, no information, other than the structure that could be derived from motion, was presented to the observer that the motion elements were not coplanar. Our experiments, however, suggest that the perceptual analysis may change if information, independent of motion, is provided to the visual system that the motion elements are in fact at different distances from the observer or, more generally, if the motion elements do not belong to the same group or partake of the same motion.

To conclude, we believe that the results of these experiments support the theory that the diagonal and vertical

\footnotetext{
${ }^{3}$ There may be a slight problem with this statement in that Gogel and Koslow (1972) claimed that there is only a small attenuation of the induced-motion effect when the dot is seen behind the frame. In our single-frame display of Experiment 1, the vertically moving dot was always perceived as behind the moving frame.
} 
effects are the consequence of the visual system's achieving a hierarchical organization among the dot, the frame, and the viewer. The visual system interprets the retinal path of the moving dot as a result of a combination of the dot's motion relative to the frame and the dot's partaking of the frame's motion. Thus the dot's motion is seen relative to the frame, and the frame's motion is perceived relative to the observer. These results also support a belongingness principle of motion perception that is analogous to a coplanarity principle of brightness perception (Gilchrist, 1977, 1979), because it is the motion of an object relative to a structure to which it "belongs," in our case a coplanar surface, that governs its perceived direction of motion.

\section{References}

Asch, S. E., \& Witkin, H. A. (1948a). Studies in space orientation I. Journal of Experimental Psychology, 38, 325-339.

Asch, S. E., \& Witkin, H. A. (1948b). Studies in space orientation II. Journal of Experimental Psychology, 38, 455-477.

Börjesson, E., \& von Hofsten, C. (1972). Spatial determinants of depth perception in two-dot motion patterns. Perception \& Psychophysics, 11, 263-268.

Brosgole, L. (1968). An analysis of induced motion. Acta Psychologica, 28, 1-44.

Brown, J. F. (1965). The visual perception of velocity. In I. M. Spigel (Ed.), Visually perceived movement (pp. 233-248). New York: Harper \& Row. (Reprinted from Psychologische Forschung, 1931, 14, 199-232)

Cutting, J. E. (1986). Perception with an eye for motion. Cambridge, MA: MIT Press.

Cutting, J. E., \& Proffitt, D. R. (1982). The minimum principle and the perception of absolute, common, and relative motions. Cognitive Psychology, 14, 211-246.

DiLorenzo, J., \& Rock, I. (1982). The rod and frame effect as a function of righting of the frame. Journal of Experimental Psychology: Human Perception and Performance, 8, 536-546.

Duncker, K. (1929). Uber induzierte Bewegung, Psychologische Forschung, 12, 180-259.

Gibson, J. J. (1979). The ecological approach to visual perception. Boston: Houghton-Mifflin.

Gilchrist, A. (1977, January 14). Perceived lightness depends on perceived spatial arrangement. Science, 195, 185-187.

Gilchrist, A. (1979). The perception of surfaces black and white. Scientific American, 240, 112-126.

Gogel, W. C., \& Koslow, M. (1971). The effect of perceived distance on induced movement. Perception \& Psychophysics, 10, 142-146.

Gogel, W. C., \& Koslow, M. (1972). The adjacency principle and induced movement. Perception \& Psychophysics, 11, 309-324.
Hochberg, J., \& Beck, J. (1954). Apparent spatial arrangement and perceived brightness. Journal of Experimental Psychology, 47, 263-266.

Hochberg, J., \& Fallon, P. (1976, December 3). Perceptual analysis of moving patterns. Science, 194, 1081-1083.

Johansson, G. (1973). Visual perception of biological motion and a model for its analysis. Penception \& Psychophysics, 14, 201211.

Johansson, G. (1975). Visual motion perception. Scientific American, 232, 76-88.

Julesz, B. (1975). Experiments in texture perception. Scientific American, 232, 34-43.

Leeuwenberg, E. L. J. (1978). Quantifications of certain visual properties: Salience, transparency, similarity. In E. L. J. Leeuwenberg \& H. F. J. M. Buffart (Eds.), Formal theories of visual perception (pp. 277-298). New York: Wiley.

Longuet-Higgins, H. C., \& Prazdny, K. (1984). The interpretation of a moving retinal image. In S. Ullman \& W. Richards (Eds.), Image understanding 1984 (pp. 179-193). Norwood, NJ: Ablex.

Marr, D. (1982). Vision: A computational investigation into the human representation of and processing of information. San Francisco: W. H. Freeman.

Restle, F. (1979). Coding theory of perception of motion configurations. Psychological Review, 86, 1-25.

Rock, I., Auster, M., Schiffman, M., \& Wheeler, D. (1980). Induced movement based on subtraction of motion from the inducing object. Joumal of Experimental Psychology: Human Penception and Performance, 6, 391-403.

Rock, I., \& Ebenholtz, S. (1959). The relational determination of perceived size. Psychological Review, 66, 387-401.

Rock, I., \& Smith, D. (1981). Alternative solutions to kinetic stimulus transformations. Journal of Experimental Psychology: Human Perception and Performance, 7, 19-29.

Shum, K. H., \& Wolford, G. L. (1983). A quantitative study of perceptual vector analysis. Perception \& Psychophysics, 34, 17-24.

Wallach, H. (1948). Brightness constancy and the nature of achromatic colors. Joumal of Experimental Psychology, 38, 310-324.

Wallach, H. (1976). On perception. New York: Quadrangle/The New York Times Book Co.

Wallach, H., Bacon, J., \& Schulman, P. (1978). Adaptation in motion perception: Alternation of induced motion. Perception \& Psychophysics, 24, 509-514.

Wallach, H., \& O'Connell, D. N. (1953). The kinetic depth effect. Joumal of Experimental Psychology, 45, 205-217.

Received October 10, 1995

Revision received June 11, 1996 Accepted July 25, 1996 


\section{ALTERNATIVE EXPLANATION OF THE DIAGONAL EFFECT}

\section{DOT'S MOTION RELATIVE TO THE SURFACE}

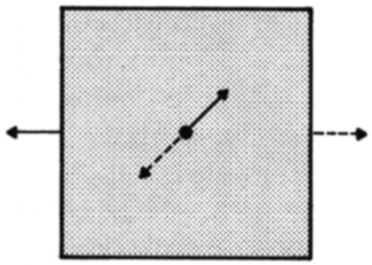

\section{RESULTANT RETINAL IMAGE}

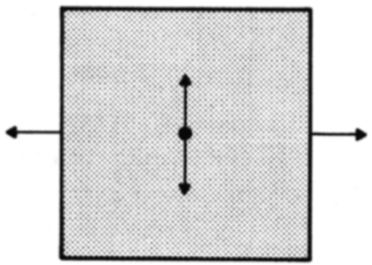

